\title{
Uso de cuplas o pares de fuerzas para expresar el torque en situaciones de equilibrio estático
}

Use of couples of forces to express torque in situations of static equilibrium

\author{
V. Slüsarenko ${ }^{1}$, R. Rojas ${ }^{*}$ \\ ${ }^{1}$ Universidad Técnica Federico Santa María, Departamento de Física, Valparaíso, Chile. \\ ${ }^{2}$ Universidad Técnica Federico Santa María, Departamento de Física, Santiago, Chile.
}

\begin{abstract}
Recibida en 12 de Noviembre, 2020. Revisado en 31 de Diciembre, 2020. Aceptado en 12 de Febrero, 2021.
Un cuerpo rígido está en equilibrio estático si se cumplen las siguientes dos condiciones: la suma de fuerzas externas es cero, y la suma de los momentos de torsión (o torques) externos es cero. La condición de equilibrio de torques presenta dificultades para algunos estudiantes porque involucra un producto cruz de vectores. Acá vamos a describir una alternativa más simple para aplicar esa condición, que consta solo de elementos escalares. Para ello acá se presentan algunos ejemplos de una varilla y de una barra en que se identifican los pares de fuerzas que producen los torques.
\end{abstract}

Palabras clave: Torque, equilibrio estático, pares de fuerzas.

A rigid body is in static equilibrium if the following two conditions are met: the sum of external forces is zero, and the sum of external torsion moments (or torques) is zero. The condition for torques turns out to be difficult for some students, because it involves a vector product. In this work we discuss an easier alternative to apply that condition, by using just scalar elements. We show some examples of a bar in static equilibrium, where we use the equilibrium of forces in order to find out the couples of forces producing torques.

Keywords: Torque, static equilibrium, couple of forces.

\section{Introducción}

La noción de torque o momento de torsión de una fuerza se define como el producto vectorial $\vec{r} \times \vec{F}$ en que $\vec{F}$ representa a la fuerza y $\vec{r}$ es la posición en que se aplica la fuerza, lo que asume la elección de un punto origen o de referencia para la posición y para el torque. Este punto de referencia puede escogerse arbitrariamente, y en la práctica uno trata de escogerlo de manera que simplifique el planteamiento de un problema. La magnitud del torque es $r F \sin \theta$ en que $\theta$ es el menor ángulo entre los vectores $\vec{r}$ y $\vec{F}$; esto permite interpretar a $r \sin \theta$ como la componente de $\vec{r}$ que es perpendicular a $\vec{F}$, y se le denomina brazo de la fuerza $(b=r \sin \theta)$.

Los textos de física que hemos revisado usan la noción de torque respecto a un punto para tratar las situaciones de equilibrio que vamos exponer acá [1, 2]. Esta noción también se usa en experiencias de laboratorio [3, en demostraciones estáticas [4, 5] en situaciones dinámicas [6, 7], en propuestas de enseñanza [8], en mediciones de aprendizaje [9] y en resolución de problemas [10]. Nosotros usaremos la magnitud $F b$ del torque y su tendencia a producir una rotación, ya sea en sentido horario o anti-horario, sin necesidad de escoger un punto de referencia ni de enfatizar el aspecto vectorial más formal del torque. Para ello vamos a identificar los pares

\footnotetext{
* Correo electrónico: roberto.rojas@usm.cl
}

de fuerzas y la distancia entre ellas, lo que corresponderá al brazo $b$. Para un cuerpo en equilibrio, la condición de que la suma de las fuerzas externas ejercidas sobre él debe ser cero, garantiza que siempre es posible distinguir pares de fuerzas de igual magnitud y direcciones opuestas. A menudo esto requiere separar una fuerza en dos partes para asociarlas a dos distintos pares de fuerza, cada uno de los cuales tendrá su propia tendencia a producir una rotación. Para ello resulta adecuado el uso de un sistema cartesiano de coordenadas y distinguir las componentes de las fuerzas en las direcciones de los ejes. Este procedimiento puede resultar más accesible para estudiantes que manifiestan dificultades para razonar con la definición del torque como un producto cruz [11] y es aplicable a situaciones de equilibrio con las fuerzas concentradas en lugares determinados.

La condición para los momentos de torsión es la que garantiza que se anula la suma de los efectos de rotación de las fuerzas aplicadas a un cuerpo. Los elementos capaces de producir una rotación sin traslación de un cuerpo son los pares de fuerzas denominados cuplas, que consisten en dos fuerzas de igual magnitud y direcciones contrarias aplicadas en puntos distantes. Un ejemplo ilustrativo es el difícil desafío de levantar una silla sujetándola con una mano por la parte inferior de una de sus patas, de modo que la silla mantenga su orientación habitual. Al aplicar una fuerza vertical igual al peso de la silla, no se lograría el objetivo porque ambas 


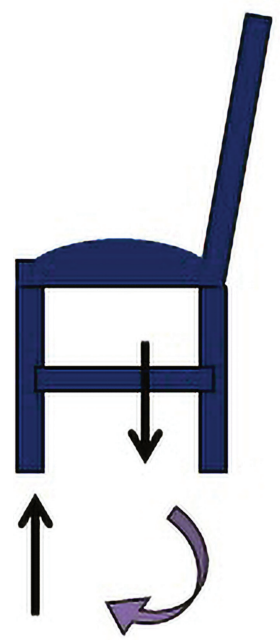

Figura 1: El peso de una silla y una fuerza vertical aplicada en una de sus patas forman una cupla que producen un efecto de rotación.

fuerzas constituyen una cupla que tiende a voltear la silla (Figura 1).

Para superar el desafío se necesita que la persona aplique además, al mismo tiempo y con una mano, un momento de torsión que anule el efecto de rotación de la cupla antes descrita. Veremos que este momento de torsión puede describirse también como una cupla.

\section{Una Varilla Horizontal}

Con el objetivo de simplificar la situación, aunque sin pérdida de generalidad, consideremos el caso en que las fuerzas se aplican a una varilla.

En primer lugar señalamos que estando la varilla sobre un plano horizontal, dos fuerzas horizontales iguales y opuestas de magnitud $F$ aplicadas a cada lado de su centro de masa, producen un efecto de rotación. Aunque la fuerza neta sobre la varilla es cero, el efecto de rotación del par de fuerzas constituye un torque neto que produce una aceleración angular, es decir, esta situación no es de equilibrio estático. En la Figura 2 se observa que estando las fuerzas a distancia $b$ producen el torque de magnitud $F b$, que es el resultado de aplicar una cupla o par de fuerzas sobre un cuerpo. Si las fuerzas no son perpendiculares a la varilla como en el caso mostrado en la Figura 2, la distancia que interviene para determinar el torque de la cupla, se debe medir entre las líneas paralelas correspondientes a las direcciones de las fuerzas.

A continuación se estudia el equilibrio de la varilla cuando se mantiene en posición horizontal, estando sujeta por un extremo y bajo el efecto de la gravedad (Figura 3).

Para ello es necesario aplicar dos fuerzas como se señala en la Figura 3 , el pulgar ejerce la fuerza $\vec{A}$ hacia arriba y el índica ejerce la fuerza $\vec{B}$ hacia abajo.

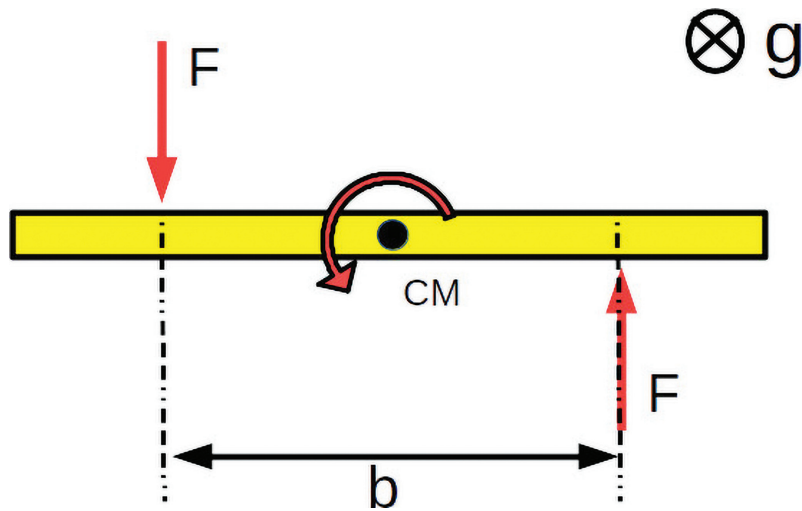

Figura 2: Varilla en un plano horizontal.

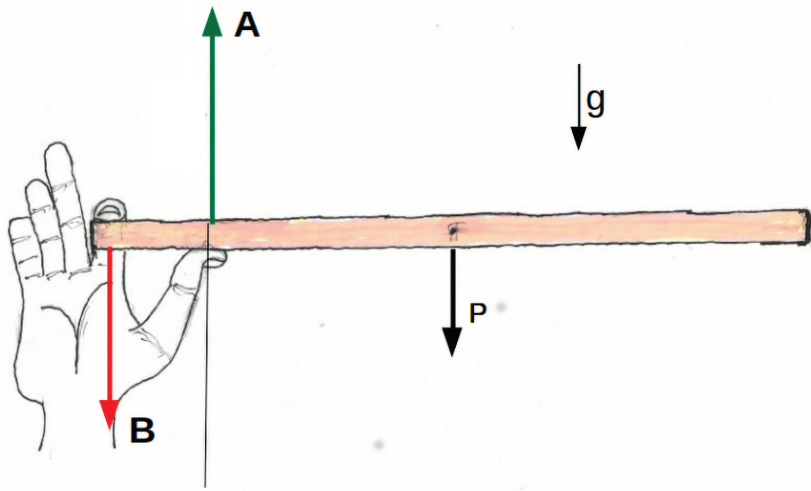

Figura 3: Varilla sujeta por un extremo, en presencia de la gravedad.

No es posible lograr el equilibrio ejerciendo solamente una fuerza en el extremo. Es sabido que, para lograr el equilibrio ejerciendo solo una fuerza, ésta debe aplicarse justo debajo del centro de masa. La fuerza $\vec{P}$ representa el peso de la varilla, y está aplicada en el centro de masa. La condición de equilibrio para la fuerzas es $\vec{A}+\vec{B}+\vec{P}=\overrightarrow{0}$, y ésta no es suficiente para determinar las fuerzas $\vec{A}$ y $\vec{B}$. Sin embargo, escribiendo $\vec{A}=-(\vec{B}+\vec{P})$ es posible distinguir dos partes en la fuerza $\vec{A}$, lo que permite interpretarlas como una fuerza hacia arriba de magnitud $P$ que forma una cupla con el peso, y otra fuerza hacia arriba de magnitud $B$ que forma una cupla con la fuerza aplicada en el extremo, como se aprecia en la Figura 4.

Según esta interpretación, con los dedos pulgar e índice de una mano se ejerce una fuerza hacia arriba de magnitud $P$, y una cupla de fuerzas de magnitud $B$ que producen un torque. Esto es similar a lo que debe hacerse con una mano en el desafío de levantar una silla. Las distancias $a$ y $b$ que aparecen en la figura permiten determinar $P b$ y $B a$ que son las magnitudes de los torques asociados a cada una de las cuplas. Ambos torques tienden a producir rotaciones en sentidos opuestos como se ilustra en la Figura 5 mediante arcos dirigidos en sentidos opuestos. Así, la condición de equilibrio de 


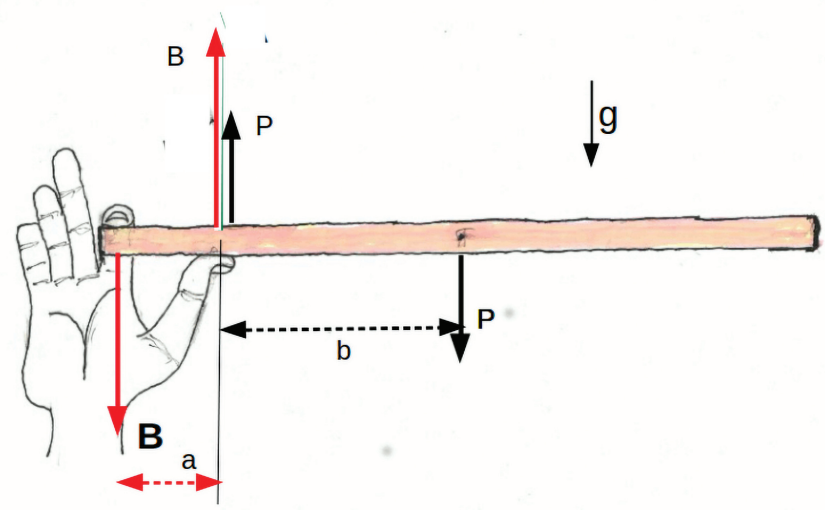

Figura 4: Aplicación del equilibrio de fuerzas y formación de cuplas.

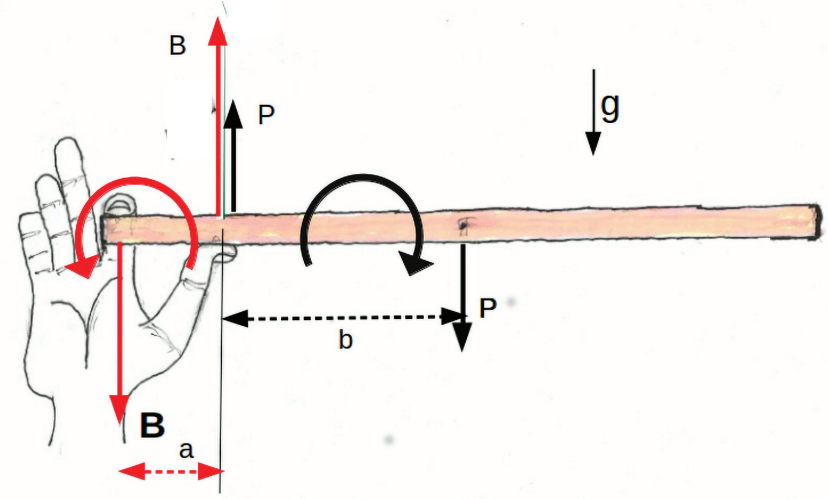

Figura 5: Torques sobre la varilla, causado por las cuplas.

torques o de momentos de torsión es $B a-P b=0$. Como es la costumbre, acá se ha asignado el signo positivo (negativo) al torque que tiende a producir una rotación en sentido anti-horario (horario).

Mediante ambas condiciones de equilibrio es posible conocer las magnitudes $A$ y $B$ en términos de la magnitud $P$ del peso y de las distancias $a$ y $b$, resultando: $A=\left(1+\frac{b}{a}\right) P$ y $B=\frac{b}{a} P$. Este resultado indica que para esta situación siempre se cumplirá que $A>B$.

Ejercicio: Se aplican a una varilla de largo $L$ las fuerzas $\vec{A}$ y $\vec{B}$ (con $A>B$ ) en sentido opuesto a los de la Figura 3, a distancia $a$ entre ellas. ¿Dónde debe aplicarse y qué valor debe tener una tercera fuerza para que la varilla quede en equilibrio?

Respuestas: La fuerza debe tener magnitud $A-B$, la distancia $b$ debe ser $b=a B /(A-B)$ y para que haya solución debe cumplirse que $A / B>L /(L-a)$.

\section{Una Barra Inclinada}

Con el objetivo de mostrar la utilidad de lo anterior en la resolución de problemas, a continuación se considera la aplicación de las cuplas para estudiar una barra de largo $L$ y peso de magnitud $P$, que se mantiene en equilibrio

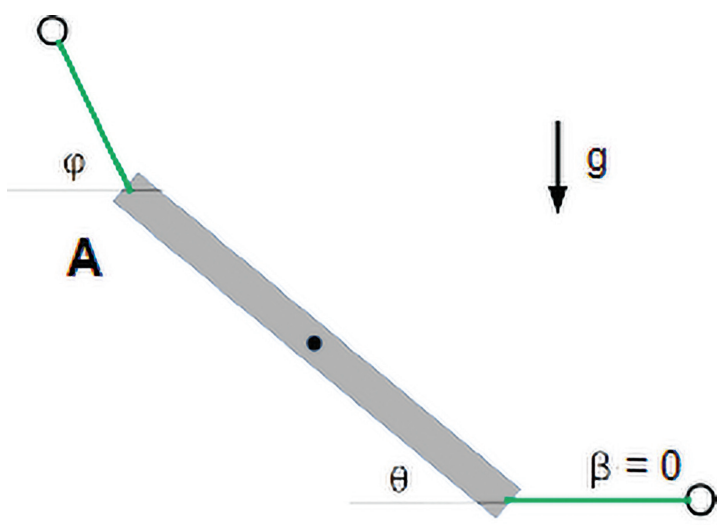

B

Figura 6: Barra inclinada en equilibrio, sujeta mediante cuerdas en ambos extremos.

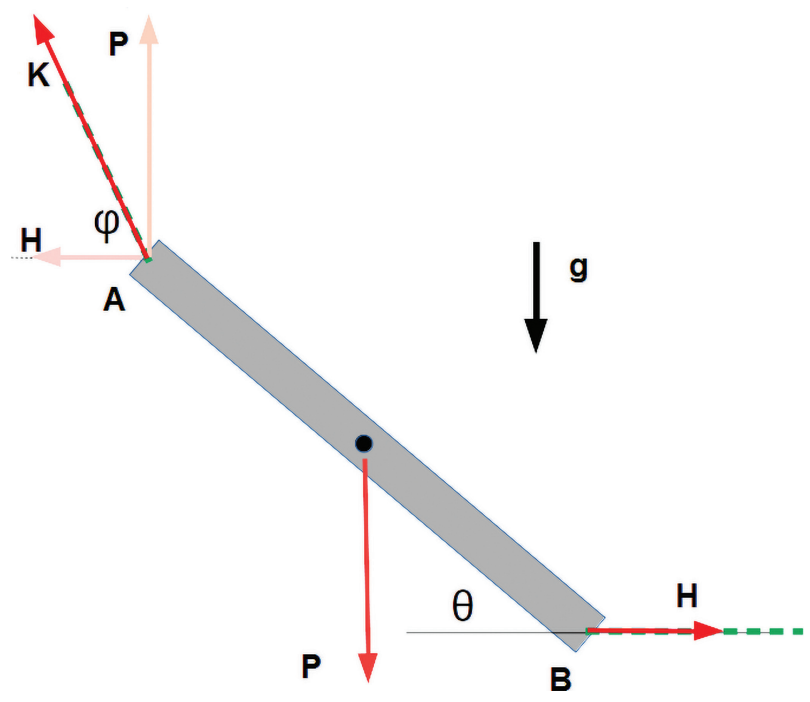

Figura 7: Barra en equilibrio mediante las fuerzas $\vec{P}, \vec{H}$ y $\vec{K}$.

formando el ángulo $\theta$ respecto a la horizontal como se muestra en la Figura 6. La barra está sujeta mediante cuerdas, una está en el extremo A y forma el ángulo $\varphi$ con la horizontal, y la otra es una cuerda horizontal que está en el extremo B. Las cuerdas tensas aplican fuerzas que tienen su misma dirección como se aprecia en la Figura 7 en que se identifica a cada una de las fuerzas.

El peso $\vec{P}$ de la barra y las fuerzas $\vec{H}$ y $\vec{K}$ que la sujetan en los extremos, deben cumplir la condición de equilibrio: $\vec{P}+\vec{H}+\vec{K}=\overrightarrow{0}$, en que la magnitud $\mathrm{P}$ es un dato y se busca obtener las magnitudes $H$ y $K$. Mediante esta relación, conviene expresar la fuerza en el extremo A por: $\vec{K}=-(\vec{P}+\vec{H})$ como se indica en la Figura 7 y utilizarla para reconocer las cuplas que producen el equilibrio de torques. La componente horizontal de $\vec{K}$ y la fuerza aplicada en el extremo B forman un par de fuerzas de magnitud $H$ que producen un torque positivo. A su vez, la componente vertical de $\vec{K}$ y el peso forman una cupla de magnitud $P$ que produce un 


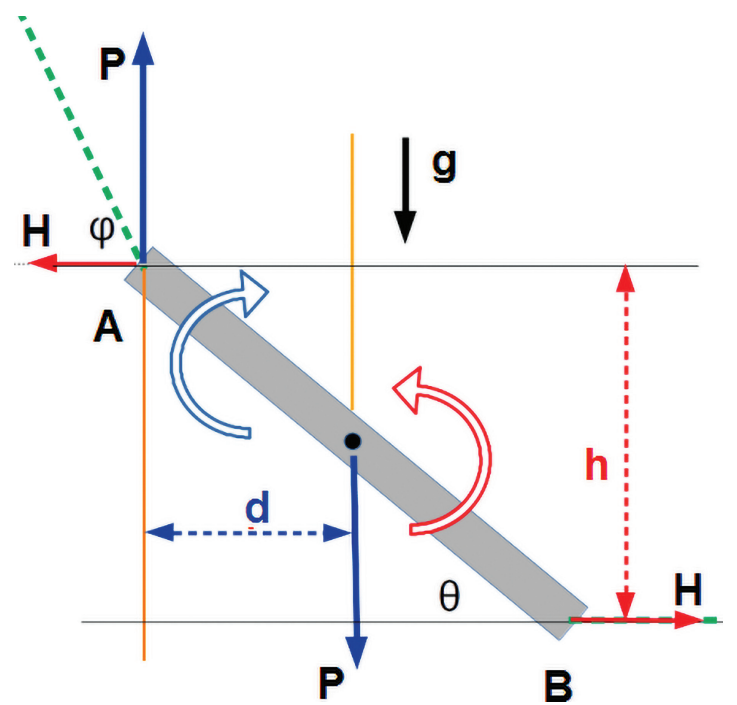

Figura 8: Barra en equilibrio de rotación por efecto de dos cuplas o pares de fuerzas.

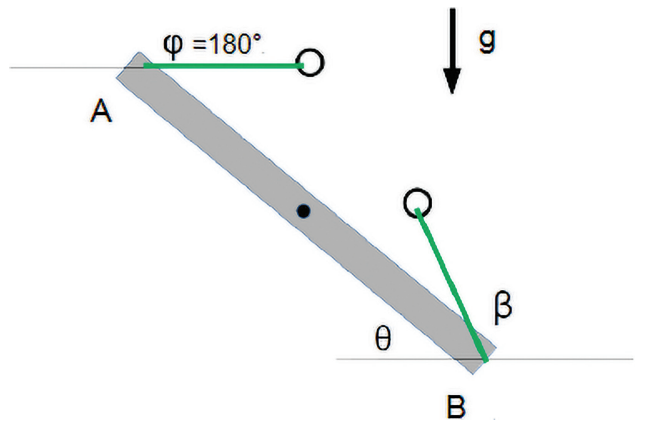

Figura 9: Barra inclinada en equilibrio, sujeta mediante cuerdas que simulan apoyos en una pared y en el suelo.

torque negativo. Ambos torques se representan en la Figura 8 mediante arcos dirigidos en sentido anti-horario y en sentido horario respectivamente, y sus magnitudes deben cumplir la condición de equilibrio: $P \frac{L}{2} \cos \theta=$ $H L \sin \theta$.

Con los datos numéricos: $M=80 \mathrm{~kg}, g \approx 10 \mathrm{~N} / \mathrm{kg}$ y $\theta \approx 53^{\circ}$, de la relación anterior se obtiene que $H=$ 300 N. Mediante las componentes de la fuerza en el extremo A, se obtiene el ángulo $\varphi$ que forma la fuerza $\vec{K}$ con la horizontal, es decir, $\tan \varphi=P / H=8 / 3$ y $\varphi=69^{\circ}$.

Otra manera de mantener una barra en equilibrio formando un ángulo $\theta$ respecto a la horizontal consiste en apoyar el extremo A en una pared vertical sin roce y el extremo B en un piso horizontal rugoso. Esta situación de equilibrio también puede lograrse mediante cuerdas colocadas en los extremos de la barra, como se muestra en la Figura 9. Al examinar las fuerzas aplicadas en cada extremo de la barra, se observa que son semejantes en magnitud y dirección a las del ejemplo anterior, pero acá son ejercidas en el extremo opuesto. Así, en el extremo A se aplica una fuerza de magnitud $H$ y en el extremo
B la fuerza aplicada tiene una componente horizontal de magnitud $H$ y una componente vertical de magnitud $P$. La consecuencia de esto es que las ecuaciones escalares de equilibrio son las mismas anteriores, y solo el torque ejercido por cada una de las cuplas acá aparece con el signo opuesto. Sin embargo esto no tiene consecuencias sobre los resultados numéricos, de modo que con los mismos datos del ejemplo anterior, en este caso también se obtienen los mismos resultados para la fuerza horizontal $\vec{H}$ y para el ángulo $\varphi$ que forma la fuerza de contacto con el suelo respecto a la horizontal.

\section{Barra con Eje Fijo}

A continuación se presenta un ejemplo más, que se distingue de los anteriores porque posee un eje fijo de rotación, que no está en un extremo ni coincide con el centro de masa, representando algo que ha sido de interés para los estudiantes. En este caso se aplica a la barra una fuerza mediante una cuerda, en el extremo más lejano al eje, con el objeto de mantenerla en equilibrio en posición horizontal, como lo muestra la Figura 10.

Para este ejemplo se usarán los siguientes datos numéricos: el ángulo de la cuerda con la horizontal es $\alpha=60^{\circ}$, el peso de la barra es de magnitud $P=400 \mathrm{~N}$ $\mathrm{y}$ las distancias son, $\overline{A B}=a=4 \mathrm{~m}, \overline{B C}=b=2 \mathrm{~m} \mathrm{y}$ $\overline{C D}=c=6 \mathrm{~m}$.

El peso $\vec{P}$ de la barra y las fuerzas $\vec{E}$ en el eje y $\vec{K}$ en el extremo deben cumplir la condición de equilibrio: $\vec{P}+\vec{E}+\vec{K}=\overrightarrow{0}$. Puesto que la fuerza $\vec{P}$ no tiene componente horizontal, la condición de equilibrio de fuerzas escrita como $\vec{P}=-(\vec{E}+\vec{K})$ muestra que las componentes horizontales de $\vec{E}$ y $\vec{K}$ deben anularse. La Figura 11 muestra la descomposición de las fuerzas $\vec{E}$ y $\vec{K}$, dónde se observa que ambas tienen componentes de magnitud $H$ en direcciones opuestas. La expresión anterior para la fuerza $\vec{P}$ también muestra que ésta puede separarse en dos partes, cada una de las cuales anula la componente vertical de las fuerzas $\vec{K}$ y $\vec{E}$, denominadas $V$ y $W$ en la Figura 11 Así, las dos fuerzas de magnitud $V$ forman una cupla que tiende a producir una rotación en sentido anti horario, y las dos fuerzas de magnitud $W$ forman otra cupla que tiende a producir una rotación en sentido horario. El efecto de rotación de tales cuplas está representado en la Figura 11 mediante arcos dirigidos en sentidos opuestos.

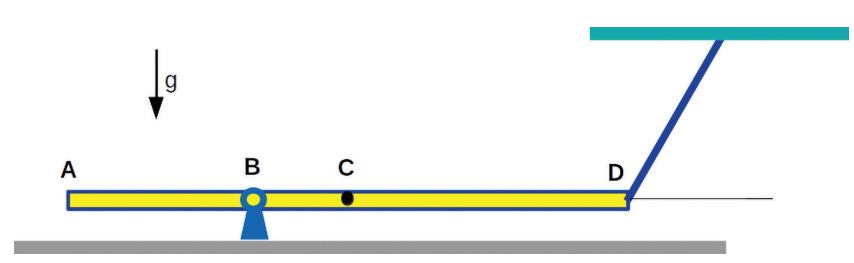

Figura 10: Barra provista de un eje a cierta distancia del centro de masa, que se mantiene en equilibrio en posición horizontal mediante una cuerda en un extremo. 


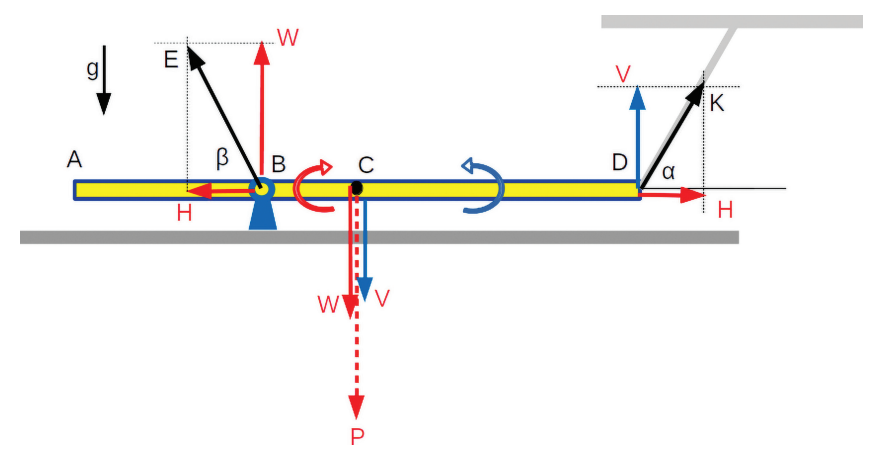

Figura 11: Descomposición de las fuerzas $\vec{P}, \vec{E}$ y $\vec{K}$ que actúan sobre la barra en equilibrio.

De acuerdo a lo representado en la Figura 11, la condición de equilibrio de torques, ejercidos por las cuplas de fuerzas de magnitudes $V$ y $W$ es $V c-W b=0$. Puesto que $P=V+W$, es posible despejar $V$ y $W$ en términos de $P$, lo que conduce a las siguientes expresiones y valores numéricos: $V=P b /(b+c)=100 \mathrm{~N}$ y $W=P c /(b+c)=300 \mathrm{~N}$. Para encontrar la magnitud $K$ de la fuerza que ejerce la cuerda se usa la relación $V=K \sin \alpha$, de lo que resulta $K=115 \mathrm{~N}$. Para la componente horizontal $H$ usamos $H=K \cos \alpha=58 \mathrm{~N}$. Con lo anterior es posible determinar la magnitud y el ángulo $\beta$ de la fuerza que ejerce el eje respecto a la horizontal. Para ello usamos $E=\sqrt{H^{2}+W^{2}}$ y $\tan \beta=W / H$, resultando $E=305 \mathrm{~N}$ y $\beta=79^{\circ}$.

\section{Discusión}

En los ejemplos de equilibrio estático que se han presentado se utiliza la condición de que la suma de fuerzas sobre la varilla o la barra debe ser cero, y a continuación se forman los pares de fuerzas de igual magnitud y dirección contraria que producen las tendencias de rotación sobre esos objetos. Para lograr lo anterior, en los ejemplos en dos dimensiones es conveniente usar un sistema cartesiano de coordenadas. Al determinar los torques se necesita obtener la distancia $b$ correspondiente a cada par de fuerzas de magnitud $F$ para hacer el producto $F b$ entre ambas magnitudes. La condición de equilibro de torques consiste en igualar o sumar algebraicamente las tendencias de rotación en sentido horario y antihorario correspondientes a los torques que producen los pares de fuerzas. Siguiendo este procedimiento se evita la elección de un punto de referencia para la posición $\vec{r}$ del punto en que se aplica la fuerza $\vec{F}$ y resulta innecesario interpretar el significado del producto vectorial $\vec{r} \times \vec{F}$, lo que evita dificultades a algunos estudiantes. Pensamos que esta manera de discutir los problemas de equilibrio estático puede ser muy bien asimilada por los estudiantes de arquitectura y ciencias de la salud, sin necesidad de entender mucho de vectores.

\section{Referencias}

[1] D. Halliday, R. Resnick y K.S. Krane, Physics (Wiley, New York, 1992), $4^{\text {th }}$ ed.

[2] D.C. Giancoli, Physics for Scientists and Engineers (Prentice Hall, Upper Saddle River, 2000), $3^{\text {rd }}$ ed.

[3] W.B. Lane, Phys. Teach. 52, 529 (2014).

[4] M. Tomac, Phys. Teach. 36, 438 (1998).

[5] I. MacInnes, Phys. Teach. 43, 538 (2005).

[6] N. Balta, Phys. Educ. 53, 013001 (2017).

[7] L. Turner y A.M. Turner, Am. J. Phys. 78, 905 (2010).

[8] W.D. Patiño Ríos, Propuesta metodológica para la enseñanza-aprendizaje del concepto de torque aplicado en palancas simples, una mirada en contexto. Tesis de Magister, Universidad Nacional de Colombia, Medellín (2015).

[9] A.B. Sarığlan y H. Küçüközer, Int. J. Phys. \& Chem. Educ. 6, 162 (2014).

[10] J. Bennett y A. Mauney, Phys. Teach. 49, 567 (2011).

[11] L.G. Ortiz, P.R.L. Heron y P.S. Shaffer, Am. J. Phys. 73, 545 (2005). 